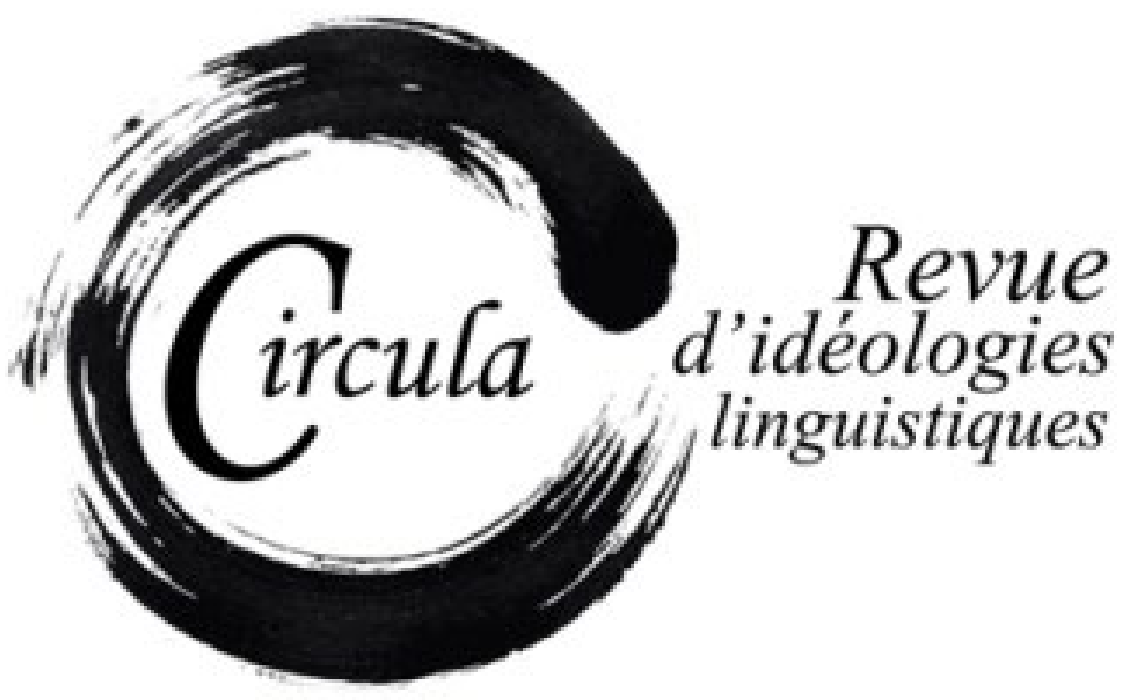

TITRE: LA RÉFORME DE L'ORTHOGRAPHE DANS LES FORUMS DE DISCUSSION DES ARTICLES DU MONDE, DU FIGARO ET DE LIBÉRATION EN LIGNE : QUE NOUS DISENT LES NON-LINGUISTES?

Auteur(s): Stefano VicARI, UniVERSitÀ di GENOVA

Revue: CirCula, NUMÉRO 4

PAgES: $106-125$

ISSN: 2369-6761

Directeurs: Wim Remysen et SABINE SchWARZE

URI: HTTP://HDL.HANDLE.NET/11143/10179

DOI: $10.17118 / 11143 / 10179$ 


\title{
La réforme de l'orthographe dans les forums de discussion des articles du Monde, du Figaro et de Libération en ligne : que nous disent les non-linguistes?
}

\author{
Stefano Vicari, Università di Genova \\ stefano.vicari@unige.it
}

Résumé : Cette étude a l'objectif d'analyser les discours métalinguistiques ordinaires circulant dans les commentaires publiés par les lecteurs du Figaro, du Monde et de Libération en ligne suite à la décision des éditeurs scolaires d'appliquer les rectifications orthographiques dans les manuels à partir de septembre 2016. L'hypothèse est que le dispositif technique dont ces journaux en ligne se sont dotés contribue à brouiller les sources énonciatives et oblige, par là, l'analyste du discours à repenser les frontières entre discours métalinguistiques « savants » et « ordinaires ». Les analyses des attitudes manifestées par les scripteurs montrent en effet que, au-delà d'un discours puriste encore largement représenté le long des débats, les espaces des commentaires en ligne des articles de journal favorisent l'hybridation de savoirs linguistiques savants et ordinaires.

Mots-clés : commentaires en ligne ; réforme de l'orthographe ; linguistique populaire ; purisme linguistique; représentations linguistiques

Abstract : This paper aims to analyze ordinary metalinguistic discourses that circulate in comments published by readers of Le Figaro, Le Monde and Liberation (online version) following the decision of schoolbooks publishers to apply the recently introduced new othography to their manuals starting from September 2016. Our hypothesis is that the technical device that these online newspapers have set up contributes to blurring enunciative sources and, therefore, it forces the discourse analyst to rethink the boundaries between "savant » and « ordinary » metalinguistic discourses. The analysis of writers' attitudes shows that, beyond a purist discourse still widely represented throughout these debates, the spaces of online commentaries to the journal articles facilitate the hybridization of scholarly and ordinary knowledge on language.

Keywords : online comments; orthography reform ; folk linguistics; prescriptive discourse ; linguistic representation 


\section{Introduction}

Dans cette étude, je me propose d'analyser les réactions des locuteurs ordinaires face à la décision de tous les éditeurs français de manuels scolaires d'appliquer les rectifications orthographiques proposées en 1990 par le Conseil supérieur de la langue française. Le 4 février 2016, une véritable querelle se déclenche, en effet, dans la presse : institutions, associations et autres voix publiques se confrontent dans ce qu'on peut à bon escient considérer comme une bataille des dires, où les différentes autorités convoquées par les journalistes argumentent, justifient, expliquent le bien-fondé de leurs positionnements face à l'entrée en vigueur de cette réforme. Qu'en est-il des locuteurs ordinaires? Dans quels espaces et sous quelles formes font-ils entendre leur voix ? Je me pencherai sur l'analyse des commentaires des locuteurs ordinaires dans les espaces interactifs des versions électroniques de trois parmi les principaux quotidiens nationaux français, à savoir Le Monde, Le Figaro et Libération, lors de la publication en ligne, le 4 février 2016, des articles suivants « Non, l'accent circonflexe ne va pas disparaître » (Le Monde) ${ }^{1}$, «Réforme de l'orthographe : ce qui change vraiment » (Libération)², "Ognon”, "nénufar", accent circonflexe : la réforme surprise de l'orthographe » (Le Figaro $)^{3}$.

Dans la suite, je me fixe l'objectif de montrer non seulement quelles sont les attitudes manifestées par les locuteurs du français face à cette prise de position, mais aussi la façon dont ces attitudes sont exprimées afin de dégager les savoirs et les connaissances métalinguistiques ordinaires et partagées par l'ensemble des scripteurs.

\subsection{La réforme de l'orthographe de 1990 à 2016 : quelques éléments de contextualisation}

Avant d'entrer dans le détail des analyses sans pourtant prétendre être exhaustif, il me semble important de rappeler quelques éléments utiles à contextualiser la décision des éditeurs français d'appliquer les recommandations de 1990.

Sans mener une analyse détaillée des trois textes journalistiques pris en compte pour cette étude, un coup d'œil aux seuls titres des articles cités plus haut suffit à saisir les attitudes, certes dissimulées, des journalistes face à cette décision des éditeurs scolaires. Si en effet Le Monde insiste à plusieurs reprises sur la continuité entre la décision des éditeurs et les rectifications proposées en 1990 et sur le poids relatif que cette décision aura sur l'orthographe du français, Le Figaro n'hésite pas à mettre en scène une véritable bataille de dires, tout en insistant sur les nouveautés entraînées par l'adoption des rectifications. Le recours à la métaphore guerrière dans le Figaro (« Toutefois les irréductibles

1. http://www.lemonde.fr/les-decodeurs/article/2016/02/04/non-l-accent-circonflexe-ne-va-pas-disparaitre_4859439_4355770.html

2. http://www.liberation.fr/france/2016/02/04/reforme-de-l-orthographe-ce-qui-change-vraiment_1431009 3. http://www.lefigaro.fr/actualite-france/2016/02/04/01016-20160204ARTFIG00080-la-reforme-de-l-orthographede-1990-fait-son-entree-dans-les-manuels-de8230-2016.php 
de l'accent circonflexe n'ont pas capitulé », «La bataille peut recommencer! ») témoigne en effet de cette exacerbation du conflit entre les positionnements adverses qui ne sont relégués que dans les toutes dernières lignes sous la plume plus modérée du journaliste de Libération. Libération semble en effet davantage insister sur la continuité entre les recommandations orthographiques de 1990 et leur adoption, aujourd'hui, par les éditeurs, ce qui est montré non seulement par le titre de l'article, mais aussi par une question rhétorique en début du dernier paragraphe : «Pourquoi a-t-on attendu aussi longtemps avant de l'appliquer? » qui permet si non de minimiser, au moins de modérer les propos des uns et des autres.

Or, s'il est vrai que dans le fond les rectifications adoptées à partir de septembre 2016 par les éditeurs sont les mêmes que celles de 1990, à cette époque-là la décision de réformer l'orthographe avait été prise suite à une longue concertation entre le Conseil supérieur de la langue française et l'Académie française, comme les trois articles n'oublient pas de le rappeler. Il s'agissait de recommandations, surtout adressées aux enseignants, et, comme le rappellent Dister et Moreau,

Les autorités se bornent à recommander aux enseignants de tenir compte des rectifications, avec plus ou moins d'insistance, avec ou sans mesures d'accompagnement [...], mais elles ne s'engagent pas sur le terrain, jugé sans doute trop aventureux, de la préparation d'une réforme plus radicale. (Dister et Moreau, 2012)

L'esprit dans lequel l'Académie avait accepté les recommandations du Conseil supérieur de la langue française était celui d'éviter toute réforme bouleversante et de se limiter à proposer quelques petits aménagements qui n'allaient pas, selon les Immortels, dans la direction d'une simplification du code écrit. Or, en février 2016, les éditeurs, sans préalable consultation avec les institutions, ont pris une décision qui entraîne l'entrée en vigueur de ces recommandations en plein régime, et par là, forceraient l'usage.

\section{La section " commentaires » des quotidiens en ligne : nouvelles géométries du discours médiatique}

\subsection{La voix "profane ": des courriers des lecteurs aux commentaires des lecteurs des quotidiens en ligne}

Plusieurs études parmi lesquelles celle menée par Paveau et Rosier (2008) ont bien montré la grande diffusion des discours métalinguistiques ordinaires; les locuteurs parlent de la langue un peu partout: des lieux institutionnels comme l'académie et l'école, aux lieux médiatiques comme la radio, la télévision et la presse, en passant par les toilettes des lieux publics et le Web. Passions et polémiques (Paveau et Rosier, 2008) se déclenchent lorsque les questions linguistiques prennent les contours de véritables débats nationaux, comme c'est le cas de la féminisation des titres et des noms de métiers (1993), de la signature de la Charte européenne des langues régionales (1998) ainsi que de la publica- 
tion du premier livre en langage « texto » (2004). Le discours médiatique s'est donc toujours emparé de ces « querelles » et n'a jamais hésité à montrer la voix des « profanes », soient-ils les lecteurs ou les participants à des émissions télévisées. Et notamment, c'est dans la presse écrite que les locuteurs peuvent depuis longtemps exprimer leur opinion sur les évènements les plus disparates à travers la rubrique « courrier des lecteurs » que les grands quotidiens nationaux ont instituée depuis belle lurette. Lieux par excellence de mise à l'épreuve du discours journalistique de la part des lecteurs, et cela de manière massive à partir des années 70 (Widart et Antoine, 2004), les courriers mettent en effet en relation les « voix médiatiques » avec le public, en ce qu'« on y mesure l'écart entre l'évènement construit par les médias et les préoccupations du public (Branca-Rosoff et Marinelli, 1994: 25). La rubrique « courrier des lecteurs » des journaux permettrait donc, pour paraphraser Branca-Rosoff et Marinelli, d'inscrire dans le débat médiatique l'auditoire qui, par là, fait entendre sa voix dans la sphère publique. Or, il me semble que les modalités de cette « inscription » dans la parole légitime du monde médiatique changent radicalement avec les forums de discussion en ligne associés aux articles publiés par les journaux. En effet, si l'on s'en tient aux études déjà menées sur les courriers des lecteurs (Branca-Rosoff et Marinelli, 1994 ; Doury et Marcoccia, 2007 ; Hubé, 2008), plus que d'une parole légitime, il vaudrait mieux parler d’une « parole légitimée » par l'équipe éditoriale qui gère la publication des courriers de manière plutôt restrictive, sur la base de critères relevant à la fois de la taille des textes, du style et, enfin, de la correspondance de la vision du monde du lecteur avec celle qui est véhiculée par le journal lui-même. La publication du courrier des lecteurs « ne doit rien au hasard, les rédactions s'ingéniant à publier les droits de réponse entre correspondants et les échos faits par ces derniers aux dossiers de presse ou à leurs éditoriaux » (Amey, 2002 : 82), alors que, pour ce qui est des commentaires des lecteurs dans les forums en ligne, comme le soutient Calabrese,

Les lecteurs occupent une place qu'aucun autre média ne leur avait accordée dans le passé. [...] Les journalistes sont attentifs et réagissent éventuellement aux commentaires des lecteurs. Ce fut le cas lors de l'événement baptisé « la révolution du jasmin » en Tunisie, où les lecteurs ont produit des arguments très élaborés pour stopper cette dénomination, considérée comme peu adéquate pour décrire la nature réelle et symbolique de l'événement. (Calabrese, 2014)

Ce type de support permet donc de modifier un tant soit peu les relations discursives entre médias et public, au moins pour ce qui est de la démarche de lecture, d'interprétation et de consommation de l'information médiatique. Comme l'affirme Calabrese (2014), les consommateurs d'informations deviennent sur le net des « prosomateurs » (consommateurs + producteurs). Si, en effet, la première source énonciative reste le journal, les lecteurs ont maintenant la possibilité de réagir aux articles au moment même de leur mise en ligne, sans qu'aucun filtre ne s'interpose, le rôle des modérateurs étant fort limité dans ce type de dispositifs (Falguères, 2007). Les lecteurs peuvent ainsi vérifier l'information par des liens hypertextuels, la rectifier ou la préciser si nécessaire, l'approfondir et la remanier en la « partageant » dans les réseaux sociaux. Le rôle du lecteur dans le jeu de la circulation 
de la parole médiatique change donc radicalement et cela grâce à des caractéristiques techniques dont les pages d'accueil des quotidiens se sont dotées.

\subsection{Le dispositif techno-discursif des forums des quotidiens en ligne : quelles retombées pour les linguistes?}

L'espace «commentaires » se trouve sur les pages d'accueil des sites des quotidiens et suit immédiatement l'article. Aux abonnés des journaux, il suffit de cliquer sur « réagir à cet article » pour publier leur propre commentaire à l'intérieur du forum consacré à l'article dont il est question sur la page elle-même. Ce dispositif techno-discursif favorise donc la libre expression du point de vue du lecteur par la simplicité de la démarche technique et permet aussi d'intégrer ce point de vue aux informations données, tout en le distinguant de l'énonciation journalistique proprement dite. Tout lecteur qui lit l'article est ainsi amené à poursuivre sa lecture jusqu'aux commentaires des autres lecteurs. L'on peut donc facilement reconnaître là un processus par lequel l'information ne suit plus exclusivement un mouvement linéaire de haut en bas, mais elle passe aussi à travers le filtre de plusieurs points de vue en confrontation. Les modalités de transmission des informations semblent bien passer d'un vers plusieurs à plusieurs vers plusieurs dans le cadre d'une démocratisation de la culture médiatique caractérisant ces dernières décennies (Durand, 1999). Pour le dire avec Calabrese, ce processus

modifie non seulement notre vision de l'information (des journalistes, du contrat de lecture qui nous lie au journal, de l'actualité, etc.), mais, en tant que linguistes, nous oblige à reformuler notre conception de ce qu'on appelle « les discours ordinaires » (en même temps que le discours spécialisé), et bien entendu leur attribution à des lieux et des rôles discursifs socialement stables. (Calabrese, 2014)

Le (socio) linguiste trouve donc là un terrain fertile pour analyser de nouvelles configurations de ce qu'on appelle communément « discours ordinaire » et des imbrications de ce type de discours avec des discours « savants », comme celui des journalistes. En effet, puisque ces supports ébranlent les frontières entre discours savant et discours ordinaire ou « profane », il faudrait mettre en lumière les procédés discursifs de co-construction des connaissances « profanes » afin de saisir la façon dont ces connaissances circulent, se diffusent, se configurent dans l'espace public et entrent en relation avec un discours de type «savant ». C'est du moins ce que je me propose de faire dans cette étude, à partir du cadre épistémologique et méthodologique de la folk linguistics (Niedzielski et Preston, 2000 ; Achard-Bayle et Paveau, 2008). Mon hypothèse de fond est que le discours métalinguistique profane ${ }^{4}$ tel qu'il se déploie dans les commentaires en ligne des lecteurs des quotidiens se caractérise par la

4. Je préfère la dénomination « discours métalinguistique profane » ou « ordinaire » à « épilinguistique », dans la suite des travaux de Rey-Debove, 1978 et Achard-Bayle et Paveau, 2008, puisque je réserve la notion d'épilinguistique pour décrire une activité inconsciente du locuteur et, qui plus est, l'emploi de cette notion sous la plume de certains linguistes implique souvent une connotation péjorative à l'égard des discours sur la langue tenus par des non-linguistes (Vicari, 2016). 
présence de positions plutôt « savantes » qui ne s'éloignent pas trop de ce qu'affirment les spécialistes de l'orthographe (voir, entre autres, Catach, 1980 ; Jaffré, 2005). Les scripteurs adopteraient en effet un degré de vigilance épistémique élevé non seulement par rapport à ce qui est écrit dans les journaux, mais aussi par rapport à ce qu'écrivent les autres participants aux fils de discussion et aux sources utilisées.

\section{Remarques sur le corpus}

Ainsi que je l'ai annoncé plus haut, j'ai choisi d'analyser le discours métalinguistique des commentaires des lecteurs de trois quotidiens nationaux en ligne (Le Monde, Le Figaro, Libération) lors de la publication simultanée de la nouvelle, le 4 février dernier, de l'adoption par tous les éditeurs scolaires des rectifications orthographiques proposées en 1990. II s'agit bien là d'un « moment discursif » (Moirand, 2007) en ce que cette nouvelle n'a pas seulement donné lieu à une abondante production au niveau médiatique le jour même de sa publication, mais aussi elle fait l'objet de plusieurs reprises et approfondissements, dans les mêmes journaux, jusqu'au mois de juin 2016 et s'inscrit dans une mémoire discursive à long terme de « querelles » sur l'orthographe du français depuis des siècles et réactivée en 1990, lors de la première proposition de rectifications (Paveau et Rosier, 2008 ; Vicari, 2016). Pour cette étude, qui a un caractère exploratoire, je me suis limité aux cinquante premiers commentaires pour chaque article publié, ce qui fait que le corpus de travail est constitué de 150 textes rédigés par des scripteurs plus ou moins ordinaires. Le choix de me limiter aux cinquante premiers textes se justifie sur la base des nombreuses études sur les échanges en ligne (voir à titre d'exemple Mangenot et Zourou, 2007) qui ont montré que la dimension interactive est souvent faible et a la tendance à s'affaiblir de plus en plus le long des échanges. Toutefois, afin de donner une idée de l'ampleur du phénomène, voici un tableau récapitulatif de l'ensemble des commentaires publiés sur les pages des différents quotidiens en ligne:

\begin{tabular}{|c|c|c|c|c|c|}
\hline & $\begin{array}{c}\text { Nombre total de } \\
\text { commentaires }\end{array}$ & $\begin{array}{c}\text { Nombre de commen- } \\
\text { taires analysés }\end{array}$ & $\begin{array}{c}\text { Nombre de } \\
\text { mots }\end{array}$ & $\begin{array}{c}\text { Date du premier } \\
\text { commentaire } \\
\text { publié }\end{array}$ & $\begin{array}{c}\text { Date du dernier } \\
\text { commentaire } \\
\text { publié }\end{array}$ \\
\hline Le Monde & 115 & 50 & 4432 & 4 février 2016 & 5 mars 2016 \\
\hline Le Figaro & 1874 & 50 & 2051 & 4 février 2016 & 29 avril 2016 \\
\hline Libération & 262 & 50 & 1601 & 4 février 2016 & 7 février 2016 \\
\hline
\end{tabular}

Tableau 1 : Total des réactions des lecteurs 
Ces quelques données montrent une grande hétérogénéité au niveau quantitatif : les lecteurs du Figaro apparaissent beaucoup plus sensibles que les lecteurs des autres quotidiens aux questions d'orthographe. En effet, non seulement les commentaires y sont plus nombreux, mais aussi ils s'étalent sur un laps de temps beaucoup plus étendu․ Cela n'a rien d'étonnant si l'on considère que Le Figaro est le seul parmi ces quotidiens qui, au moins dans sa version papier, publie périodiquement une rubrique consacrée aux questions linguistiques, «Le bon français », tenue par des écrivains, des journalistes ou des académiciens et à propos de laquelle Ayres-Bennet (2015) a remarqué de nombreuses affinités avec le discours des remarqueurs de la langue française. L'on est donc en droit d'avancer l'hypothèse que le lectorat du Figaro est particulièrement sensible aux questions linguistiques et qu'il s'identifie davantage avec un public de « bons locuteurs » du français, en l'occurrence conservateur en matière de langue. Cela dit, il faut aussi considérer que Le Monde consacre aux questions linguistiques un blog "Langue sauce piquante », où les correcteurs et les lecteurs interagissent sur les aspects les plus disparates de la langue française. C'est ainsi que le même jour, le 4 février, les correcteurs publient un billet sur la décision des éditeurs français d'adopter les rectifications orthographiques et les nombreuses réactions des lecteurs (au nombre de 123) ne tardent pas.

Toutefois, l'hétérogénéité du corpus se manifeste également au niveau de la taille des commentaires et des identités des scripteurs. On peut en effet facilement constater que la longueur des textes est très variable d'un journal à l'autre, les lecteurs du Monde étant en moyenne plus prolixes que les autres. Comme les analyses qualitatives le montreront du moins partiellement, les commentaires de Libération se limitent à un seul, parfois deux énoncés, alors que les scripteurs du Monde et du Figaro articulent de manière plus analytique leurs raisonnements.

En ce qui concerne les identités des scripteurs, les pseudonymes garantissant un anonymat du moins partiel, il est impossible d'élaborer un profil sociolinguistique précis des scripteurs. Or, non seulement cette situation semble caractériser la plupart des échanges en ligne entre locuteurs, au-delà des pages des quotidiens analysées, mais elle en représente un trait constitutif et permet, entre autres, la présence simultanée de locuteurs ayant différents niveaux de « spécialisation » dans les différentes matières traitées. La prise en compte de ce brouillage énonciatif se révèle donc essentielle pour comprendre le fonctionnement de ces discours en ligne et oblige à repenser les frontières entre savoirs savants et savoirs populaires : tout locuteur peut en effet avoir accès à un grand nombre d'articles et d'ouvrages scientifiques et de vulgarisation qui lui permettent d'augmenter ses stan-

\footnotetext{
5. Il faut néanmoins remarquer qu'un simple coup d'œil aux commentaires des internautes portant sur d'autres articles issus de différentes rubriques (politique étrangère, société, éco, culture, sports) montre les mêmes tendances au niveau du nombre de réactions publiées: dans Le Figaro les commentaires sont en moyenne beaucoup plus nombreux que dans les deux autres journaux alors que Libération est sans aucun doute le quotidien en ligne qui présente le moindre nombre de textes rédigés par ses lecteurs.
} 
dards épistémiques ${ }^{6}$, il peut interagir avec d'autres « pairs » dans le cadre interactionnel qu'offrent les échanges sur les forums et, par là, demander des conseils, résoudre des doutes, etc. :

L'idée même de «communauté épistémique » ou de « réseau sociocognitif » c'est-à-dire d'un réseau d'agents partageant des croyances et des normes épistémiques dont la structure est déterminée par l'interaction entre agents et en même temps influence la distribution des connaissances - se concrétise sur Internet d'une façon bien plus transparente que dans d’autres réseaux sociaux. (Origgi, 2006)

Dès lors, les échanges en ligne constituent un lieu privilégié pour observer la manière où les connaissances plus ou moins ordinaires se co-construisent dans les interactions, se propagent dans les différentes communautés et permettent de repenser les identités discursives en termes de fluidité et d'abandon des rôles discursifs stables.

\section{Les positionnements face à l'adoption des rectifications : quelques données quantitatives}

Avant d'entrer dans le détail des analyses qualitatives, voici un tableau des différents positionnements exprimés par les scripteurs face à la décision des éditeurs d'adopter les rectifications orthographiques. Outre les attitudes favorables et contraires à l'adoption de la réforme de l'orthographe, j'ai repéré des commentaires où la position du scripteur n'est pas explicitée, celui-ci se limitant à répondre à ses interlocuteurs sur des questions ponctuelles issues des discussions.

\begin{tabular}{|c|c|c|c|}
\hline & Pour & Contre & On ne sait pas \\
\hline \multirow{2}{*}{ Le Monde } & 27 & 20 & 3 \\
& $54 \%$ & $40 \%$ & $6 \%$ \\
\hline \multirow{2}{*}{ Le Figaro } & 4 & 46 & 0 \\
\hline \multirow{2}{*}{ Libération } & $8 \%$ & $92 \%$ & $0 \%$ \\
\hline Total & 11 & 14 & $50 \%$ \\
\hline & $22 \%$ & $28 \%$ & 28 \\
& 52 & 80 & $19 \%$ \\
\hline
\end{tabular}

Tableau 2 : Les attitudes des lecteurs face à la réforme de l'orthographe

6. Certes, le fait d'avoir potentiellement accès à des savoirs scientifiques ne constitue pas une condition suffissante pour que les locuteurs ordinaires les reconnaissent comme des autorités fiables et, par là, que ces savoirs puissent circuler au-delà des milieux scientifiques. Néanmoins, il est indéniable que le développement du Web a radicalement modifié la manière d'accès aux savoirs tant du point de vue de la richesse des informations que du point de vue des potentialités communicatives. Pour un traitement plus approfondi de cette question, voir Origgi (2006). 
Tout d'abord, ce tableau montre une forte polarisation des positions, surtout dans Le Monde et dans Le Figaro. Ce dernier se caractérise en effet par l'absence de commentaires où les scripteurs n'explicitent pas leur point de vue. Il en va autrement pour les lecteurs de Libération qui, si l'on se tient aux données, semblent traiter de cette question sans pourtant prendre des positions explicites. Ensuite, si plus que la moitié des lecteurs du Monde expriment un jugement positif face à la réforme de l'orthographe, le public du Figaro semble, du moins à première vue, plutôt renfermé sur des positions puristes, les attitudes favorables à une régularisation des particularités de l'orthographe étant presque absentes.

Néanmoins, les pourcentages concernant l'ensemble des commentaires montrent que plus d'un tiers des scripteurs accueille avec faveur la nouvelle de l'adoption des rectifications, ce qui constitue une donnée intéressante si l'on pense que la « défense » de l'orthographe constitue l'un des piliers du discours puriste en France depuis longtemps (Paveau et Rosier, 2008). Or, bien que la présence d'attitudes plutôt réticentes à toute innovation dans le domaine de l'orthographe apparaisse comme majoritaire, ces données permettent d'avancer l'hypothèse, à confirmer par les analyses qualitatives suivantes, que le discours métalinguistique ordinaire a quelque peu évolué et s'aligne un tant soit peu sur ce que les spécialistes de l'orthographe soutiennent depuis une trentaine d'années lorsqu'ils prônent une réforme timide de l'orthographe (Catach, 1980 ; Jaffré et Fayol, 1997).

\section{De quelques arguments contre la réforme de l'orthographe}

La plupart des positions des scripteurs contraires aux modifications orthographiques rentrent de plein droit dans le discours puriste autour de l'orthographe française, qui a déjà fait l'objet de plusieurs analyses ponctuelles (entre autres Paveau et Rosier, 2008 ; Paveau, 2008) et d'enquêtes approfondies plus ou moins récentes (Millet, Lucci et Billiez, 1990 ; Dister et Moreau, 2012 : 197). Ces études ont bien mis en évidence les représentations partagées autour de ce niveau de l'analyse linguistique et en ont dénoncé les idéologies sous-jacentes. Ces représentations révèlent un certain immobilisme linguistique et font des pièges et des difficultés de l'orthographe des beautés auxquelles il semble impossible de renoncer, au risque de dénaturer la langue française. C'est ainsi qu'arguments esthétiques, éthiques, linguistiques et sociologiques (l'argument étymologique, l'association hâtive entre capacités intellectuelles et maîtrise de l'orthographe, la notion de faute et ses conséquences, le nivellement par le bas, le niveau d'études et le discours décliniste sur les pratiques langagières contemporaines, etc.) se combinent dans un imaginaire de l'orthographe plutôt rétif à toute réforme, celle-ci étant souvent considérée comme une atteinte grave à la clarté, à la pureté et à la beauté de la langue française, comme le montrent ces quelques commentaires issus du corpus Figaro:

7. Je ne m'attarderai donc pas sur l'analyse de ce genre de commentaires, ces études ayant été menées sur des corpus plus larges et plus représentatifs que le mien. Je renvoie donc à ces recherches pour des synthèses plus approfondies. 
L’orthographe sophistiquée était le luxe de notre chère langue. Par elle, notre exigence baroque de la beauté et de l'emphase transparaissait dès le premier mot. Autant enlever les gargouilles de Notre-Dame qui ne signifient plus rien aujourd'hui ! Cette loi est un abandon du patrimoine. (ena phobe, le 04 février 2016 - 10:34, Le Figaro) ${ }^{8}$

Bonjour. Quelle réforme? Quand on lit la prose des rédacteurs, le français est massacré depuis déjà longtemps. Voulue en 1990, cette réforme appliquée 26 ans plus tard laissera bien à nos chères têtes blondes encore quelques 80 mots de vocabulaire pour s'exprimer d'ici une vingtaine d'années. S'exprimer, pas forcément se comprendre. (Bacbuc, le 04 février 2016 - 10:44, Le Figaro)

L'assimilation de la langue avec un monument historique, le discours décliniste qui considère la simplification orthographique comme un symptôme du déclin généralisé de la langue à l'heure actuelle ainsi que l'idée selon laquelle la réforme de l'orthographe entraînerait une réduction du lexique du français constituent des « prédiscours » (Paveau, 2006) largement répandus auprès des locuteurs et se fondent sur l'inscription en discours d'une communion de sentiments et de valeurs présumés partagés par tous. La prégnance de ces prédiscours est montrée par le fait que même là où les commentaires montrent une attitude plutôt ambigüe face à la simplification orthographique, ils s'appuient sur eux pour s'en détacher, comme cela arrive dans le commentaire suivant :

Avez vu lu le texte de la réforme de 1990 ? Je n'ai, quant à moi, pas très bien compris le nivellement par le bas qu'elle réalisait. évènement / événement ou aimè-je / aimé-je. Ce n’est pas très clair. Les classes supérieures, instruites et distinguées écriraient événement ou aimé-je tandis que les basses classes, peu instruites, bas de gamme pour parler comme PMM écriraient évènement ou aimè-je? . On voit les effets dévastateurs du passage de l'accent aigu à l'accent grave. (Jean Leonguy, le 07 février 2016 - 12h35, Le Monde)

Le classement social effectué sur la base de la maîtrise de l'orthographe est alors dénoncé par ce scripteur qui montre l'absurdité, à ses yeux, d'une telle démarche. Ce texte, tout comme beaucoup d'autres dans le corpus, exemplifie bien ce que Paveau a appelé « théorie négative » des prédiscours, qui se manifeste à travers une posture de «filtrage cognitif » c'est-à-dire une « attitude qui vise à éradiquer toute manifestation des prédiscours dans ses propres productions, au nom d'un idéal de la table rase » et de " procès cognitif » qui consisterait à exprimer « des commentaires dénonciatifs et/ou accusateurs du discours de l'autre » (Paveau, 2006 : 138).

8. Aucune correction n’a été apportée aux textes du corpus. 
Or, si les attitudes contre tout type d'intervention en matière d'orthographe sont majoritaires, une analyse qualitative plus fine des positions contraires montre qu'il arrive souvent que l'opposition à la réforme ne rentre pas forcément dans une critique puriste de tout type d'évolution. En effet, dans une quinzaine de commentaires (soit presque $19 \%$ des jugements contraires à la réforme dans le corpus), les scripteurs expriment leur désaccord face à la réforme telle qu'elle a été conçue ou proposée.

L'évolution de l'orthographe est un fait historique. La langue Française évolue, ordonnance de Villers-Cotterêt, Académie Française de Richelieu, etc. Ce qui me chiffonne par contre c'est la simplification en raison non pas d'une amélioration linguistique mais plutôt du fait de la perte de la langue, de la question du gender et d'autres aspects boboïsants. (Galvatron, le 4 février 2016 - 10:20, Le Figaro)

Bonjour, « réformette », réforme sur des détails, peu déterminants. Orthographe d’un mot, est elle fonction des « racines » Grecs ou latines, ou du erreur de reproduction « du Moyen age »? Par contre, la vraie simplification est occultée, par exemple, un « S» au pluriel; et ce pour tous les mots qui se terminent par un « $U$ » : un clou, des clous, un bijou, des .. bijous. Etc .. (Lovyves -e, le 4 février 2016 - 10:30, Le Figaro)

Ce ne sont pas mes oignons, mais mais le bulletin officiel des nouveaux ignares aurait mieux fait de s'attaquer, par exemple, à la règle du pluriel de certains mots en « al » (royaux mais fatals), ou adjectifs qui sont perclus d'exceptions « bisous mais bijoux » etc... Je veux bien nénufar, mais acceptons «farmacie » («farmacia » en italien) etc.. (Babillage, le 4 février 2016 - 11:20, Le Figaro)

L'orthographe française est à l'écriture ce que le code du travail est à la législation : une usine à gaz. Ce n'est pas d'une petite réformette (dont l'application n'est même pas obligatoire) qu'il nous faut, mais d'une réforme traumatisante où l'on sait qu'il y avait un « avant » et un « après qu'il nous faut, un peu comme les turcs ont fait leur réforme sous ATATURK, en non seulement réformant leur orthographe, mais en plus en changeant d'alphabet (et en turkisant un grand nombre de mots venant de l'arabe ou du persan). On se plaint que le français a du mal à lutter contre l'emprise de l'anglais, mais on se refuse à lui donner les outils pour se défendre. Les petits réajustements annoncés vont bien sûr dans le bon sens, nénuphar vient de l'iranien et donc on ne voit pas pourquoi on mettrait un ph, mais pourquoi ne pas s'inspirer de nos voisins italiens, espagnols, portugais, qui écrivent Pharmacie avec un F (farmacia), qui tous ont fait leur réforme (certains d'ailleurs en sont à leur $5^{\circ}$ réforme) sans que cela ne les traumatise particulièrement. (alainblainFeatured, le 4 février 2016 - 14:17, Libération)

C'est très étonnant cette réforme de 1990. En la laissant facultative, on a rendu l'orthographe encore plus complexe en voulant la simplifier. Et que dire de ces mots féminisés qu'on veut désormais nous imposer; les auteures, professeures, et autres écrivaines? Et les associations LGTB qui écrivent simplifié-e-s? (Maxiton, le 4 février 2016 - 11:27, Le Figaro) 
On est bien loin là de l'image d'une orthographe intouchable et immuable: sous la plume de ces scripteurs non seulement l'orthographe devrait être réformée, mais cette réforme devrait aller plus loin dans la régularisation des incohérences et toucher, par exemple, les pluriels irréguliers et les lettres parasites étymologiques qui font partie, d'ailleurs, des zones fragiles de l'orthographe du français (Jaffré et Fayol, 1997).

\section{Pourquoi réformer? Ce que nous disent les non-linguistes}

\subsection{Evolution historique de l'orthographe : l'exemple des grands auteurs}

La raison la plus répandue dans le corpus en faveur de l'adoption de la réforme est représentée par l'argument de l'évolution de l'orthographe. Les scripteurs insèrent donc la réforme dans le cadre plus vaste de l'évolution historique des conventions orthographiques et, pour ce faire, ils s'appuient volontiers sur l'autorité des grands auteurs:

C'est l'ordre politique qui se manifeste dans le travail normatif sur la langue. L'idéologie de l'Ancien Régime a visé à briser les solidarités linguistiques régionales entre le peuple et l'aristocratie, d'où la « latinisation » parfois erronée de l'orthographe, à l'usage des courtisans en représentation devant le roi. Ainsi « poids » (de « pensum », corrigé d'après « pondus »). Même Vaugelas écrivait: « Une erondelle ne fait pas le printans. » Joli, non? (zadig, le 4 février 2016 - 13h49, Le Monde)

« lls m’ont nommé de la cadémie. Cela m'irai comme une bage à un cha » Maréchal de Richelieu (XVIIIème siècle). « Dans l'affreux cimetière... fleurit le nénuphar » V. Hugo (en "Châtiments », sur l'air de Malbrouk). « Je m'en vais ou je m'en vas - l'un et l'autre se dit ou se disent » dernières paroles attribuées à Vaugelas, greffier du bon usage au XVIlème siècle. Voir aussi les «tolérances orthographiques» de 1902. Transmis par J.M. Mathieu. (NICOLAS DRUI, le 4 février 2016 - 16h11, Le Monde)

@peio2b Avez vous lu Montaigne? je ne pense pas. (michel92, le 4 février 2016 - 15:37, Libération)

Vaugelas, Richelieu, Montaigne et Hugo sont ainsi convoqués par les scripteurs en tant que figures patrimoniales non seulement de la langue, mais aussi de la culture française. L'inscription en discours de cet ensemble de bons auteurs garantit au scripteur «son adéquation aux valeurs, aux fondements d'une collectivité » (Maingueneau, 2004 : 112) et, par là, fonctionne en guise de justification des dires. Cela est montré également par le fait que ces auteurs sont placés dans une posture de surénonciation (Rabatel, 2004) par le biais du discours rapporté direct entouré d'un discours d'escorte valorisant les dires cités. Toutefois, il ne suffit pas de citer ces auteurs pour que les dires soient automatiquement cautionnés, il faut les mentionner « correctement ", ce qui est montré par l'échange suivant, où l'on assiste à une sorte de réduction de la portée de la citation d'Orwell : 
Orwell était vraiment un visionnaire! D’abord la Novlangue a été généralisée au nom du «politiquement correct », nommant par exemple « ministère de la Défense »le ministère de la guerre, ou « dommages collatéraux » les civils innocents victimes de bavures militaires. Ensuite on « simplifie » la langue, pour que peu à peu, entre l'abrutissement télévisuel, l'acculturation médiatique et la « simplification » plus aucune idée complexe ou nuancée ne puisse être exprimée. C'est bien triste futur que nous préparons à nos enfants... (Tyler-Durden, le Featured, le 4 février 2016 - 15:32, Libération)

@Tyler-Durden Cette réforme n'a rien voir avec la novlangue d'Orwell. Dans 1984 il s'agissait de raccourcir les mots et d'en former de nouveaux par des mots composés afin de réduire la pensée. (bruno75, le 4 février 2016 - 16:06, Libération)

La critique généralisée des pratiques langagières contemporaines menée par le premier scripteur sur la base de la présumée clairvoyance d'Orwell est aussitôt estompée, sinon contredite, par la re-contextualisation des positions de l'auteur de 1984. Dans cet échange, ce n'est pas vraiment l'autorité du grand auteur qui est critiquée, mais plutôt son réemploi pour soutenir un positionnement adverse à la réforme. Le rôle patrimonial des « pères » de la langue n'est pas mis en discussion, tant il est vrai que leur mention, toujours en surénonciation, sert à ridiculiser les propos des opposants à la réforme:

Vous savez sans doute que nous devons à l'Almanach Vermot la disparition de l'imparfait et du plus que parfait du subjonctif sauf à la 3e personne du singulier. Nous attendons donc de vous voir jouir dans un prochain post en utilisant les 2 temps de ce mode. (Jean Leonguy, le 4 février 2016 - 18h11, Le Monde)

J'aime ces commentaires réactionnaires et caricaturaux, qui font avancer le débat. Je ne sais pas ce qu'est un français oral (ou écrit) pur. Une langue est éminemment plastique, sans quoi nous parlerions toujours le latin de Tacite. Ce qui, je vous le concède, ne manquerait pas d'élégance. Par ailleurs, votre post comporte trois fautes : «À quand » et non pas «A quand »; «(Dans » et non pas «( Dans »; « négligée !) » et non pas «négligée!) ). Même les posts au Monde méritent du soin... Lol mdr! (Grand naïf, le 4 février 2016 - 14h15, Le Monde)

Loin de se renfermer dans une vision idyllique d'un passé mythique où la langue française aurait connu son apogée, comme souvent cela arrive dans le discours puriste (Vicari, 2016), l'appel aux pères de la langue fonctionne dans ces textes à l'instar d'une preuve considérée comme évidente et objective en faveur d'une vision de l'orthographe en évolution, et permet d'inscrire en discours une communauté de valeurs partagées par les locuteurs du français. 


\subsection{Les autres langues ont réformé : l'italien et l'allemand comme modèles à suivre}

Le deuxième argument le plus répandu parmi les partisans de la réforme tient à la comparaison avec des langues voisines, telles que l'italien, l'allemand et l'espagnol. Cette comparaison peut être établie sur des aspects ponctuels, comme la perte, en italien, de ph:

Les italiens ont simplifié leur orthographe au début du siècle dernier. Photographe s'écrit fotografo avec pour principe « on écrit ce que l’on prononce ». II ne s'en porte pas plus mal. La complexité de l'orthographe française était une protection corporatiste des clercs contre la plèbe, maintenant on en fait un trésor national. (Nicolas, le 4 février 2016 - 14h19, Le Monde)

L'exemple italien a alors l'objectif de montrer que l'adoption du principe phonographique (« on écrit ce que l'on prononce »), du moins dans le cas des lettres parasites, n’aurait pas de conséquences néfastes sur la langue : en effet, le maintien des lettres étymologiques ne serait que le résultat, d'après le scripteur, de la volonté d'une classe de perpétrer des inégalités sociales contre les plus faibles. Or, selon certains spécialistes de l'orthographe, les lettres étymologiques et historiques font partie d'

une zone floue, instable, voire anarchique. On n'en finirait pas d'énumérer les exceptions, les anomalies, concernant des graphies incorporées pour des raisons aujourd'hui sans intérêt, et qui ne subsistent que comme traces des choix graphiques, plus ou moins heureux, ou comme erreurs et incohérences malheureuses d'une époque antérieure. (Cogis, 2005 : 51).

Le commentaire de ce scripteur a donc au moins le mérite d'identifier l'une des zones instables de l'orthographe, c'est-à-dire « [...] des zones de fragilité, définies comme particulièrement propices à l'occurrence de variations non conformes aux conventions » (Jaffré et Fayol, 1997 : 102) qui seraient indépendantes des scripteurs et des contextes. La comparaison avec les autres langues peut aussi se faire à un niveau plus global, comme dans les commentaires suivants:

S'inspirer de l'approche allemande : de l'autre côté du Rhin l'orthographe a été privatisée, ce qui fait que chaque éditeur de livres, journaux, etc. applique ses règles maison. Résultat des courses : ceux qui s'adressent à des lecteurs raffinés continuent à proposer une orthographe raffinée, ceux qui s'adressent à des lecteurs plus... basiques proposent une orthographe plus basique, et tout le monde est content, les vaches sont bien gardées, l'orthographe ne fait plus événement. (Pat Cartier 04/02/2016 - 20h22, Le Monde)

Pourquoi l'italien qui est d'origine latine tout comme le français est si simple (grammaire, orthographe) et nous nous avons pris un malin plaisir à complexifier tout cela.... Le français se voulait la langue du peuple et elle lui a été volée pour montrer aux pauvres gents qu'ils étaient incultes. Du coté italien, pas de pays réunifié jusqu'à voici 150 ans. La langue est restée au peuple et elle est de facto devenue simple \& compréhensible par tous. Ahhh ce besoin pédantesque de nos Elites (jn 04/02/2016 - 18h13, Le Monde) 
@kock @alainblain comme les italiens, les espagnols, les allemands, (xufusu-616@tmpFeatured 5 Février 2016 à 2:30, Libération)

La question sur laquelle ces derniers scripteurs insistent est liée au rôle joué par les représentations de l'orthographe en France, qui en font un moyen de classement social des locuteurs : la mise en perspective historique avec les autres pays permet donc de relativiser son poids et de dénoncer les inégalités sociales qui dérivent d'une importance démesurée accordée aux bonnes pratiques orthographiques. C'est du moins ce que montrent certains commentaires du corpus, où la mauvaise maîtrise de l'orthographe aurait des conséquences négatives dans le monde du travail :

Ça ne changera rien à la nullité crasse des élèves qui font de toute façon 15 fautes par page et qui savent très bien que le bac n'est pas un enjeu puisqu'il leur est offert par l'Education nationale. (Jeanne Oliphant Le 04/02/2016 à 10:31, Le Figaro)

\subsection{Eliminer les éléments « inutiles »: signes suprasegmentaux et homophones}

Le dernier argument par lequel les scripteurs soutiennent le projet de réforme tient à la présumée inutilité de certains signes suprasegmentaux et à la nécessité d’apporter quelques simplifications dans le cadre des homophones. En voici des exemples:

En quoi le gouter est il moins savoureux que le goûter ?? Son cout serait plus conforme s'il était un coût, parait-il !! En quoi l'orthographe est pervertie, la France ridicule et la société au bord du gouffre si son orthographe simplifie quelques aberrations linguistiques ?? Connaitre les exceptions incohérentes et inutiles de la langue peut faire paraitre plus intelligent mais ce n'est que pédanterie !! l'orthographe n'est que la science des ânes !! (Francois Michel, le 04 février 2016 - 15h29, Le Monde)

Réformer l'orthographe ne serait pas anecdotique. Que de temps et d'énergie perdus pour l'orthographe ! La lettre « e », par exemple, peut se prononcer d'une dizaine de manières différentes... Chaque son peut s'écrire de trop nombreuses manières... Ce n'est pas une réforme de l'orthographe qu'il nous faut, mais une révolution! Cela libèrerait énormément de temps pour des apprentissages bien plus utiles. (KMT, 04 février 2016 - 16h26, Le Monde)

La langue est au service de la pensée et de ses échanges, une langue qui n'évolue pas est morte. J'ai fait du latin jusqu'en terminale et je ne le regrette pas, mais je trouve que des irrégularités qui n'affinent pas le sens du contenu méritent d'être simplifiées. Le mauvais usage systématique de la grammaire me semble beaucoup plus grave car il révèle deux incapacités: accepter des règles et les comprendre - pensée confuse d'égos surdimensionnés (Phil, 04 février 2016 - 22h20, Le Monde) 
Cette réforme de l'accent circonflexe comme celui de l'accord du verbe laisser devant un infinitif ne me parait pas scandaleuse. On a laissé son accent là où il était nécessaire et c'est bien là le principal. Par exemple est-il nécessaire de mettre un accent circonflexe à brûler alors que brume n'en prend pas; ou à traîner alors que drainer n'en prend pas? (bruno75, 04 février 2016 - 15:12, Libération)

Comme on peut le constater, ces critiques à l'orthographe telle qu'elle est pratiquée jusqu'aujourd'hui portent sur la régularisation de l'emploi de l'accent circonflexe et sur la simplification de la question des homophones. On retrouve donc, encore une fois sous la plume de ces scripteurs, des propos qui ne s'éloignent pas beaucoup de ce que nous disent les spécialistes de l'orthographe déjà cités ci-dessus. La simplification des incongruités de l'orthographe du français permettrait en outre de faciliter l'apprentissage du code écrit, comme le soutiennent les scripteurs du corpus:

l'orthographe... la science des imbéciles : ce serait assez drôle si çà nous avais pas couté tellement en temps, argent, et échecs pédagogiques. Enfin bon... maintenant, c'est bien trop tard de toute façon, un gamin intelligent qui voit que des jeunes diplômés ne bossent pas, vous lui ferez surtout pas perdre son temps avec çà... (jbl92, le 04 Février 2016 - 15:22, Libération)

Surtout qu'en leur faisant apprendre ces règles archaïques, on leur faisait se persuader qu'en tant que natifs ils n'étaient pas détenteur de l'utilisation de leur langue maternelle. (Clément Michard, le 04/02/2016 - 10:44, Le Figaro)

Les scripteurs dénoncent par là le rôle que l'école a réservé à l'apprentissage d'une orthographe qui, nous disent-ils, est riche en pièges et en difficultés considérées comme inutiles. Le dernier scripteur pointe notamment l'insécurité linguistique que ces pièges auraient provoquée auprès des apprenants. 


\section{Conclusion}

Les échanges en ligne autour des articles des quotidiens semblent confirmer du moins partiellement l'hypothèse énoncée en début de cette étude concernant l'hybridation de savoirs profanes et savants. S'il est vrai que dans les commentaires analysés les scripteurs ne citent pas ou ne s'appuient jamais sur l'autorité des textes scientifiques, l'on constate néanmoins qu'un bon nombre de positions ne diffèrent pas trop des positions des scientifiques en matière d'orthographe.

De nombreux scripteurs montrent en effet non seulement des attitudes plutôt favorables à l'adoption d'une réforme, mais aussi ils vont jusqu'à identifier des «zones » instables et à proposer des solutions, bien que partielles et fondées sur des prédiscours ordinaires. Les dispositifs techno-discursifs sur les pages des journaux semblent donc non seulement modifier la façon dont le lecteur a accès aux informations mais aussi participer de la création d'espaces de dialogue à l'intérieur desquels des usagers plus ou moins savants ou ordinaires peuvent se confronter et ouvrir des débats féconds sur les questions linguistiques les plus variées. II me parait donc que le réseau Internet et que ce type de dispositifs invitent en particulier les analystes du discours à repenser les frontières nettes entre savoirs savants et savoirs populaires en faveur d'un continuum de discours des plus aux moins ordinaires et à essayer de mieux cerner les modalités à travers lesquelles les connaissances métalinguistiques se diffusent dans les nouvelles formes de participation collaborative en ligne. 


\section{Références}

Achard-bayle, Guy et Marie-Anne Paveau (dir.) (2008), Linguistique populaire ?, numéro thématique de Pratiques, vol. 2, nº 139-140.

Ayres-Bennett, Wendy (2015), «La persistance de l'idéologie linguistique des remarqueurs dans les chroniques de langage de 1925 à nos jours », Circula, n 1, p. 44-68.

Branca-Rosoff, Sonia et Cécile Marinelli (1994), «Faire entendre sa voix : le courrier des lecteurs dans les trois quotidiens marseillais », Mots, $n^{\circ}$ 40, p. 7-24.

Calabrese, Laura (2014), "Rectifier le discours d'information médiatique : quelle légitimité pour le discours profane dans la presse d'information en ligne? », Les Carnets du Cediscor, n 12, disponible sur http://cediscor.revues.org/916 [Page consultée le 15 juin 2016].

Catach, Nina (1980), L'orthographe du français, Paris, Nathan.

Cogis, Danièle (2005), Pour enseigner et apprendre l'orthographe, Paris, Delagrave.

Dister, Anne et Marie-Louise Moreau (2012), Réforme de l'orthographe française : craintes, attentes et réactions des citoyens, numéro thématique de Glottopol, n 19, disponible sur http://glottopol. univ-rouen.fr/numero_19.html [Page consultée le 7 décembre 2016].

Doury, Marianne et Michel Marcoccia (2007), « Forum internet et courrier des lecteurs : l'expression publique des opinions », Hermès, n 47, p.41-50.

Durand, Pascal (1999), «La “culture médiatique” au XIXème siècle. Essai de définition-périodisation », Quaderni, n³9, p. 29-40.

Falguères, Sophie (2007), «Les forums de discussion des sites web de la presse quotidienne? », dans Dahmani Ahmed (dir.), La démocratie à l'épreuve de la société numérique, Paris, Karthala, disponible sur http://loiseaugerard.free.fr/DELcolloque/DEL,\%20d\%E9lib\%E9ration/DEL\%20 12,\%20Falgu\%E8res.pdf [Page consultée le 10 juin 2016].

Hubé, Nicolas (2008), «Le courrier des lecteurs : une parole journalistique profane ? », Mots : les langages du politique, n 87, disponible sur http://mots.revues.org/12572. [Page consultée le 10 juin 2016].

Jaffré, Jean-Pierre et Michel Fayol (1997), L'orthographe, des systèmes aux usages, Paris, Flammarion.

Jaffré, Jean-Pierre (2005), «L'orthographe est un château-fort: commentaires sur l'enquête du collectif "sauver les lettres" », disponible sur www.cafepedagogique.net [Page consultée le 10 juin 2016].

Maingueneau, Dominique (2004), «Hyperénonciateur et “particitation” », Langages, n 156, p. 111126.

Mangenot, François et Katerina Zourou (2007), « Susciter le dialogue interculturel en ligne : rôle et limites des tâches », Lidil, n³ 36, p. 43-68. 
Millet, Agnès et Vincent Lucci et Jacqueline Billiez (1990), Orthographe mon amour!, Grenoble, Presses Universitaires de Grenoble.

Moirand, Sophie (2007), Les discours de la presse quotidienne : observer, analyser, comprendre, Paris, Presses Universitaires de France.

Niedzielski, Nancy et Denis Preston (2000), Folk linguistics, Berlin, New York, De Gruyter.

Origgi, Gloria (2006), « Autorité épistémique et internet scientifique : la diffusion du savoir sur internet », disponible sur http://jeannicod.ccsd.cnrs.fr/ijn_00000678/en/ [Page consultée le 10 juin 2016].

Paveau, Marie-Anne (2006), Les prédiscours : sens, mémoire, cognition, Paris, Presses de la Sorbonne Nouvelle.

Paveau, Marie-Anne (2008), « Chronique "linguistique". Les accents : fonction linguistique ou passion orthographique? ", Le français aujourd'hui, no 160, p. 103-110.

Paveau, Marie-Anne et Laurence Rosier (2008), La langue française : passions et polémiques, Paris, Vuibert.

Rabatel, Alain (2004), «l'effacement énonciatif dans les discours rapportés et ses effets pragmatiques », Langages, n 154, p. 3-17.

Rey-Debove, Josette (1978), Le métalangage, Paris, Le Robert.

Rosier, Laurence (2004), "La circulation des discours à la lumière de "'effacement énonciatif" : l'exemple du discours puriste sur la langue », Langages, no 156, p. 65-78.

Vicari, Stefano (2016), Pour une approche de la linguistique populaire en France : attitudes, prédiscours, questions de confiance, Rome, Aracne.

Widart, Marie et Frédéric Antoine (2004), « Regard sur l'évolution historique du courrier des lecteurs dans la presse », Recherches en communication, n²1, disponible sur http://sites.uclouvain.be/ rec/index.php/rec/article/view/4631/4361 [Page consultée le 10 juin 2016]. 